12

\title{
Влияние адсорбции водорода на трансформацию Стоуна-Уэльса в углеродных нанотрубках малого диаметра
}

\author{
(С) Л.А. Опенов ${ }^{1}$, А.И. Подливаев ${ }^{1,2}$ \\ ${ }^{1}$ Национальный исследовательский ядерный университет „МИФИ“, \\ Москва, Россия \\ ${ }^{2}$ Научно-исследовательский институт проблем развития научно-образовательного потенциала молодежи, \\ Москва, Россия \\ E-mail: laopenov@gmail.com
}

(Поступила в Редакцию 23 октября 2017 г.)

В рамках неортогональной модели сильной связи изучено влияние гидрирования углеродных нанотрубок $(4,0)$ и $(3,0)$ на трансформацию Стоуна-Уэльса. Показано, что адсорбция атомарного водорода может приводить как к понижению, так и к повышению барьеров для прямой и обратной трансформации в зависимости от ориентации поворачивающейся связи С-C относительно оси нанотрубки. Сделана оценка характерных времен образования и отжига дефектов Стоуна-Уэльса. Рассчитаны модули Юнга.

Работа поддержана грантом РФФИ № 18-02-00278-а и выполнена при поддержке Министерства образования и науки РФ в рамках Программы повышения конкурентоспособности НИЯУ МИФИ.

DOI: $10.21883 /$ FTT.2018.04.45695.302

\section{1. Введение}

В низкоразмерных углеродных материалах (фуллеренах [1], нанотрубках [2], графене [3]) самым распространенным типом дефектов являются топологические дефекты Стоуна-Уэльса (Stone-Wales, SW). Они образуются при трансформациях $\mathrm{SW}$ - поворотах связей $\mathrm{C}-\mathrm{C}$ на угол $\approx 90^{\circ}[4]$. В результате каждого такого поворота изменяются размеры нескольких соседних углеродных колец. Так, если поворачивающаяся связь $\mathrm{C}-\mathrm{C}$ является общей для двух 6-атомных колец в графене, то в результате трансформации SW появляются два 5-атомных и два 7-атомных кольца.

В углеродных наноструктурах трансформации SW и дефекты SW могут играть различную роль. Например, бакминстерфуллерен $\mathrm{C}_{60}$ с симметрией $I_{h}[1]$ образуется, по-видимому, из исходно разупорядоченного кластера $\mathrm{C}_{60}$ путем цепочки обратных трансформаций SW [5]. Дефекты SW в углеродных нанотрубках (УНТ), с одной стороны, уменьшают их механическую жесткость [6,7], a с другой - могут улучшить функциональные характеристики УНТ, поскольку являются центрами предпочтительной адсорбции различных химических элементов $[8,9]$. В графене дефекты SW влияют на механические свойства, электронную структуру и фононные спектры [10,11]. Рассеивая электронные волны, они снижают подвижность носителей заряда.

Дефекты SW образуются, как правило, на стадии изготовления той или иной наноструктуры. Кроме того, они возникают при облучении образца частицами с высокой энергией (ионами, электронами и т.п.), а также при сильной механической деформации [12]. Чтобы очистить наноструктуру от дефектов SW можно, например, нагреть ее до температуры, при которой тепловая энергия достаточна для отжига дефектов [13].
Интенсивность формирования и отжига дефектов SW определяется высотой соответствующих активационных барьеров на поверхности потенциальной энергии (potential energy surface, PES). Ранее было показано [14], что в фуллерене $\mathrm{C}_{60}$ эти барьеры можно существенно понизить путем адсорбции на кластер атомарного водорода. Аналогичный результат получен при исследовании адсорбции водорода и молекул ОН на графен [15] (см. также [16]).

Цель настоящей работы заключается в компьютерном моделировании прямой и обратной трансформации SW в гидрированных одностенных УНТ. Для определенности мы ограничиваемся рассмотрением адсорбции водорода только на внешней стороне УНТ, т.е. считаем, что в полость УНТ водород не проникает. Такая ситуация имеет место в очень узких УНТ. При увеличении диаметра УНТ внешнее гидрирование становится энергетически невыгодным (например, в УНТ типа $(n, 0)$, начиная с $n=5[17])$. По этой причине в настоящей работе изучены одни из самых узких УНТ, $(4,0)$ и $(3,0)$, наряду с их полностью гидрированными производными $(4,0) \mathrm{H}$ и $(3,0) \mathrm{H}$ со стехиометрией $\mathrm{C}: \mathrm{H}=1: 1$. Мы сопоставляем характеристики дефектов SW в гидрированных и негидрированных УНТ, включая энергии формирования, активационные барьеры и частотные факторы, а также делаем оценку времени образования и отжига дефектов при нескольких температурах. Для всех рассмотренных УНТ рассчитан модуль Юнга.

\section{2. Методы расчета}

Мы моделировали УНТ $(4,0)$ и $(3,0)$ сверхъячейками $\mathrm{C}_{160}$ и $\mathrm{C}_{120}$ соответственно, каждая из которых включает 
10 элементарных ячеек и имеет длину около $4 \mathrm{~nm}$, что превышает характерный размер области деформации создаваемой дефектом SW в УНТ [18]. Для моделирования УНТ $(4,0) \mathrm{H}$ и $(3,0) \mathrm{H}$ использовались сверхъячейки $\mathrm{C}_{160} \mathrm{H}_{160}$ и $\mathrm{C}_{120} \mathrm{H}_{120}$ соответственно. Граничные условия выбирались периодическими в направлении оси УНТ и свободными в двух поперечных направлениях. Период сверхъячейки всякий раз находился из условия минимума энергии равновесной конфигурации.

Энергия формирования дефекта SW определялась как разность энергий УНТ с дефектом и без него: $E_{f}=E_{\mathrm{SW}}-E_{0}$. Для расчета высоты $U_{f}$ активационного барьера, препятствующего образованию дефекта, мы находили энергию $E_{\mathrm{TS}}$ переходного состояния (transition state), которое является седловой точкой PES первого порядка (т.е. имеет в спектре собственных колебаний одну мнимую частоту). При этом $U_{f}=E_{\mathrm{TS}}-E_{0}$, а высота барьера, препятствующего отжигу дефекта, равна $U_{a}=E_{\mathrm{TS}}-E_{\mathrm{SW}}$. Частотные факторы $A_{f}$ и $A_{a}$ для образования и отжига дефекта SW определялись по формуле Виньярда [19] на основании расчета спектров собственных колебаний в соответствующих стационарных точках PES.

Межатомные взаимодействия $\mathrm{C}-\mathrm{C}, \mathrm{C}-\mathrm{H}$ и $\mathrm{H}-\mathrm{H}$ описывались в рамках неортогональной модели сильной связи [20], которая в явном виде включает квантовомеханический („зонный“) вклад электронной подсистемы в полную энергию и учитывает все валентные электроны: по одному от каждого атома водорода $(1 s)$ и по четыре от каждого атома углерода $(2 s$ и $2 p)$. Этот подход продемонстрировал свою применимость к численному моделированию широкого круга углеродных и углеводородных наноструктур (см. работы $[13,16,21-24]$ и ссылки в них).

\section{3. Результаты и их обсуждение}

3.1. Нанотрубки $(4,0)$ и $(4,0) \mathrm{H}$. В УНТ $(n, 0)$ имеется два типа неэквивалентных связей $\mathrm{C}-\mathrm{C}$ : параллельные оси УНТ и направленные под углом к ней. Дефекты SW, которые образуются при поворотах этих связей, будем называть дефектами SWA и SWB соответственно (см. рис. 1). Для энергии формирования дефектов SWA в УНT $(4,0)$ мы получили $E_{f}=0.47 \mathrm{eV}$, тогда как $E_{f}=2.61 \mathrm{eV}$ для дефектов SWB. Эти величины меньше рассчитанных нами в рамках той же модели для УНТ $(10,0)[13](3.30$ и $3.47 \mathrm{eV})$ в соответствии с общей тенденцией к понижению $E_{f}$ при уменьшении диаметра УНТ [25].

Анализ зависимости энергии УНТ от угла поворота связи $\mathrm{C}-\mathrm{C}$ при трансформации $\mathrm{SW}$ позволяет определить высоты энергетических барьеров $U_{f}$ и $U_{a}$, препятствующих соответственно образованию и отжигу дефекта. Мы получили $U_{f}=2.63 \mathrm{eV}, U_{a}=2.16 \mathrm{eV}$ для дефекта SWA (рис. 2) и $U_{f}=6.35 \mathrm{eV}, U_{a}=3.74 \mathrm{eV}$ для дефекта SWB. Частотные факторы $A_{f}$ и $A_{a}$, которые

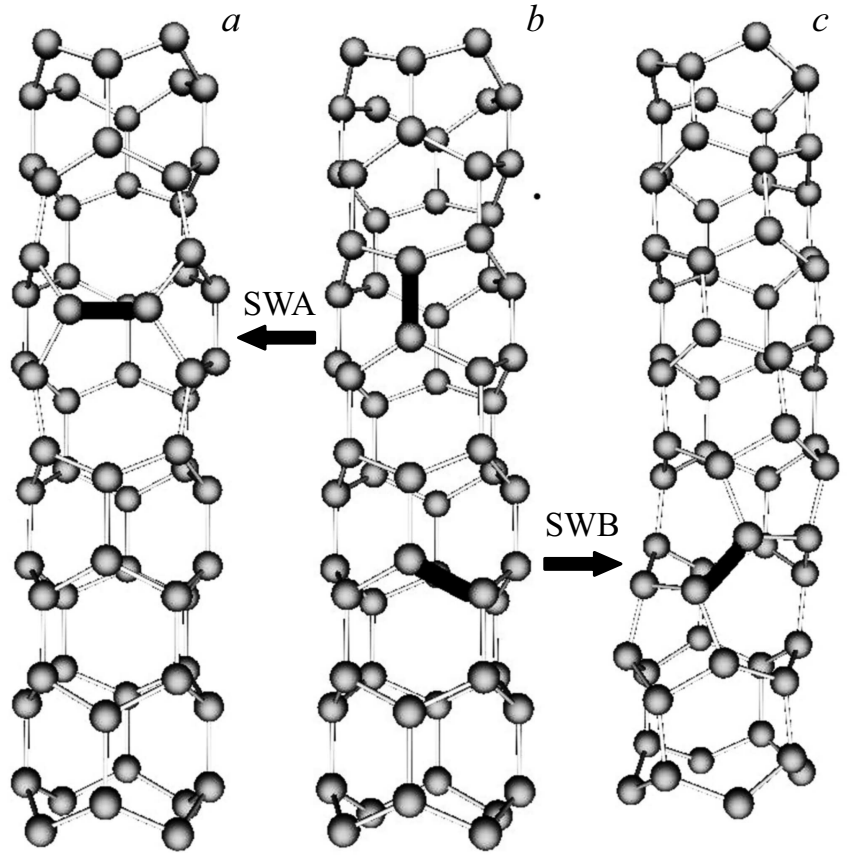

Рис. 1. Образование дефектов SWA (слева) и SWB (справа) на примере нанотрубки $(4,0)$. Поворачивающиеся при трансформациях SW связи C-C выделены черным цветом.

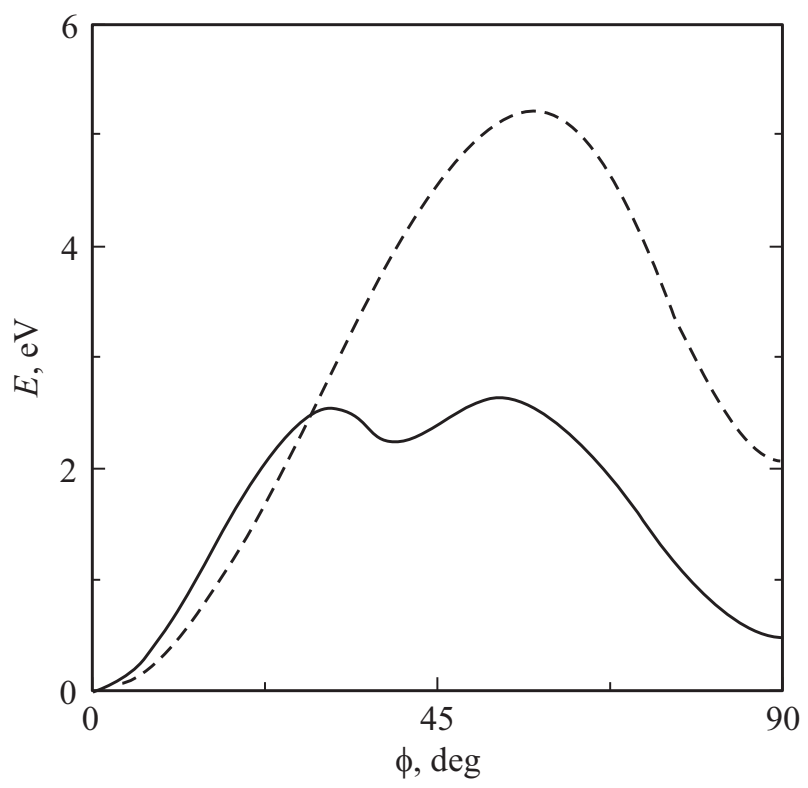

Рис. 2. Зависимости энергий $E$ нанотрубок $(4,0)$ (сплошная линия) и $(4,0) \mathrm{H}$ (штриховая линия) от угла $\phi$ поворота связи $\mathrm{C}-\mathrm{C}$ при образовании дефекта SWA (см. рис. 1). За начало отсчета в обоих случаях принята энергия $E(\phi=0)$ соответствующей бездефектной УНТ.

наряду с высотами соответствующих барьеров определяют интенсивность образования и отжига дефектов, составляют $A_{f}=4.15 \cdot 10^{13} \mathrm{~s}^{-1}, A_{a}=1.59 \cdot 10^{15} \mathrm{~s}^{-1}$ для дефектов SWA и $A_{f}=1.92 \cdot 10^{13} \mathrm{~s}^{-1}, A_{a}=4.01 \cdot 10^{14} \mathrm{~s}^{-1}$ для дефектов SWB. 
Характерное время $\tau$ протекания того или иного термоактивированного процесса можно найти, используя закон Аррениуса:

$$
\frac{1}{\tau(T)}=A \exp \left[-\frac{E_{a}}{k_{\mathrm{B}} T}\right],
$$

где $A$ - частотный фактор этого процесса, $E_{a}-$ его энергия активации, $k_{\mathrm{B}}$ - постоянная Больцмана. Полагая энергию активации равной высоте соответствующего энергетического барьера, для времени образования дефектов SWA и SWB найдем соответственно $\tau \sim 0.1 \mu \mathrm{s}$ и $\sim 10 \min$ при $T=2000 \mathrm{~K}, \sim 10 \mu \mathrm{s}$ и $\sim 10^{3}$ days при $T=1500 \mathrm{~K}$ и т.д., тогда как для времени отжига этих дефектов получим $\tau \sim 0.1 \mathrm{~ns}$ и $\sim 10 \mu$ s при $T=2000 \mathrm{~K}$, $\sim 10 \mathrm{~ns}$ и $\sim 10 \mathrm{~ms}$ при $T=1500 \mathrm{~K}$ и т.д. (понижение температуры приводит к экспоненциальному росту всех этих времен).

В гидрированных УНТ $(4,0) \mathrm{H}$ связи С-C при трансформациях SW поворачиваются вместе с адсорбированными на них атомами водорода. Энергии формирования дефектов SWA и SWB равны $E_{f}=2.06$ и $1.10 \mathrm{eV}$ соответственно. Энергетические барьеры составляют $U_{f}=5.22 \mathrm{eV}, U_{a}=3.15 \mathrm{eV}$ для дефекта SWA (рис. 2) и $U_{f}=4.38 \mathrm{eV}, U_{a}=3.28 \mathrm{eV}$ для дефекта SWB. Таким образом, при гидрировании УНТ барьеры для образования и отжига дефектов SW могут как увеличиваться (SWA), так и уменьшаться (SWB). Частотные факторы $A_{f}=1.65 \cdot 10^{17} \mathrm{~s}^{-1}, A_{a}=1.42 \cdot 10^{17} \mathrm{~s}^{-1}$ для дефектов SWA и $A_{f}=6.58 \cdot 10^{15} \mathrm{~s}^{-1}, A_{a}=2.05 \cdot 10^{15} \mathrm{~s}^{-1}$ для дефектов SWB. Оценка по формуле (1) дает для времени образования дефектов SWA и SWB соответственно $\tau \sim 0.1 \mathrm{~ms}$ и $\sim 10 \mu$ s при $T=2000 \mathrm{~K}, \sim 2 \mathrm{~s} \mathrm{и} \sim 0.1 \mathrm{~s}$ при $T=1500 \mathrm{~K}$ и т.д., а для времени отжига этих дефектов $\tau \sim 1 \mathrm{~ns}$ и $\sim 0.1 \mu \mathrm{s}$ при $T=2000 \mathrm{~K}, \sim 0.1 \mu \mathrm{s}$ и $\sim 0.1 \mathrm{~ms}$ при $T=1500 \mathrm{~K}$ и т.д., то есть характеристическое время увеличивается (уменьшается) в соответствии с повышением (понижением) активационных барьеров.

3.2. Нанотрубки $(3,0)$ и $(3,0)$ Н . Известно, что с термодинамической точки зрения УНТ $(3,0)$ неустойчивы относительно соответствующих фрагментов графена [26]. Одним из следствий такой неустойчивости является отрицательная величина энергии формирования дефектов SWA в этих УНT, для которой мы нашли $E_{f}=-0.56 \mathrm{eV}$, т.е. при трансформации SW возникает энергетически более выгодная конфигурация. Энергия формирования дефектов SWB, напротив, положительна и составляет $E_{f}=3.26 \mathrm{eV}$ - больше, чем в УНТ $(4,0)$. Барьеры для образования и отжига дефектов SWB равны $U_{f}=6.39 \mathrm{eV}$ и $U_{a}=3.13 \mathrm{eV}$, а частотные факторы $A_{f}=2.98 \cdot 10^{16} \mathrm{~s}^{-1}$ и $A_{a}=4.59 \cdot 10^{15} \mathrm{~s}^{-1}$ соответственно. Для времени образования и отжига найдем соответственно $\tau \sim 1 \mathrm{~s}$ и $\sim 10 \mathrm{~ns}$ при $T=2000 \mathrm{~K}, \sim 1$ dау и $\sim 10 \mu$ s при $T=1500 \mathrm{~K}$ и т.д.

Гидрированные УНТ $(3,0) Н$ термодинамически устойчивы [17]. Они интересны тем, что в результате ряда трансформаций SW из этих УНТ получаются ал- мазоподобные нанонитки (nanothreads) [27,28], в которых повернутые связи $\mathrm{C}-\mathrm{C}$ „перегораживают“ полость УНТ, приводя к формированию уникальных наноструктур, отличных и от УНТ, и от обычных полимеров. В УНТ $(3,0) \mathrm{H}$ энергии формирования обоих типов дефектов SW положительны. Они составляют $E_{f}=1.22$ и $0.92 \mathrm{eV}$ для дефектов SWA и SWB соответственно. Энергетические барьеры и частотные факторы равны $U_{f}=4.36 \mathrm{eV}, U_{a}=3.14 \mathrm{eV}$, $A_{f}=1.36 \cdot 10^{17} \mathrm{~s}^{-1}, A_{a}=1.51 \cdot 10^{17} \mathrm{~s}^{-1}$ для дефектов SWA и $U_{f}=4.24 \mathrm{eV}, U_{a}=3.32 \mathrm{eV}, A_{f}=1.47 \cdot 10^{16} \mathrm{~s}^{-1}$, $A_{a}=3.44 \cdot 10^{15} \mathrm{~s}^{-1}$ для дефектов SWB. Оценка времен образования дефектов SWA и SWB по формуле (1) дает соответственно $\tau \sim 1$ и $\sim 3 \mu$ s при $T=2000 \mathrm{~K}, \sim 3$ и $\sim 10 \mathrm{~ms}$ при $T=1500 \mathrm{~K}$ и т.д., тогда как времена отжига этих дефектов имеют порядок $\tau \sim 1 \mathrm{~ns}$ и $\sim 0.1 \mu \mathrm{s}$ при $T=2000 \mathrm{~K}, \sim 0.1$ и $\sim 30 \mu \mathrm{s}$ при $T=1500 \mathrm{~K}$ и т.д.

Заметим, что гидрирование УНТ $(3,0)$ приводит к весьма существенному понижению барьера для образования дефектов SWB. Именно эти дефекты являются важным элементом структуры алмазоподобных нанониток $[27,28]$, формируя пары соседних дефектов, расположенные по длине УНТ случайным образом.

3.3. Механическая жесткость. С целью сравнения механической жесткости различных УНТ, для каждой из них мы рассчитали величину модуля Юнга, который (по аналогии с трехмерными и двумерными материалами [29]) находили по формуле

$$
Y_{1 D}=L^{-1}\left(\partial^{2} V / \partial \varepsilon^{2}\right)_{\varepsilon \rightarrow 0}
$$

где $L-$ длина УНТ, $V$ - ее полная энергия, $\varepsilon-$ относительная деформация растяжения вдоль оси УНТ. При таком подходе модуль Юнга измеряется в ньютонах, а результат не зависит от эффективной площади $S$ поперечного сечения УНТ, в определении которой имеется известный произвол $[17,30]$.

Для УНТ $(4,0)$ мы нашли $Y_{1 D}=310 \mathrm{nN}$. Полагая величину $S$ равной площади кольца шириной $3.35 \AA$ (расстояние между соседними слоями в графите), со-

Модули Юнга $Y_{1 D}$ негидрированных и гидрированных нанотрубок $(4,0)$ и $(3,0)$ с различными дефектами SW

\begin{tabular}{c|c|c}
\hline Нанотрубка & Дефект & $Y_{1 D}, \mathrm{nN}$ \\
\hline$(4,0)$ & Без дефектов & 310 \\
$(4,0)$ & SWA & 279 \\
$(4,0)$ & SWB & 281 \\
$(4,0)$ & Без дефектов & 242 \\
$(4,0) \mathrm{H}$ & SWA & 226 \\
$(4,0) \mathrm{H}$ & SWB & 224 \\
$(3,0)$ & Без дефектов & 221 \\
$(3,0)$ & SWA & 165 \\
$(3,0)$ & SWB & 177 \\
$(3,0) \mathrm{H}$ & Без дефектов & 195 \\
$(3,0) \mathrm{H}$ & SWA & 150 \\
$(3,0) \mathrm{H}$ & SWB & 170
\end{tabular}


держащего периметр УНТ [30,31], получим в привычных единицах $Y=Y_{1 D} / S=870 \mathrm{GPa}$, что согласуется с типичными для одностенных УНТ экспериментальными значениями $Y \sim 1 \mathrm{TPa}[32]$. Для УНТ $(3,0)$ мы получили $Y_{1 D}=221 \mathrm{nN}$.

Результаты для этих и других УНТ представлены в таблице. Видно, что полное гидрирование УНТ $(4,0)$ и $(3,0)$ приводит к уменьшению $Y_{1 D}$. Это объясняется тем, что в гидрированных УНТ связи С-С между $s p^{3}$-гибридизованными атомами углерода менее прочные, чем связи между $s p^{2}$-гибридизованными атомами в негидрированных УНТ. Авторы работы [17] пришли к выводу, что гидрированные УНТ, напротив, являются более жесткими. Их расчеты основаны на ином определении величины $S$ - исходя из длины элементарной ячейки УНТ и объема, приходящегося на один атом в алмазе.

Жесткость УНТ с дефектами SW меньше, чем у бездефектных УНТ. Это согласуется с данными других авторов [6,7] и справедливо как для негидрированных, так и для гидрированных УНТ.

\section{4. Заключение}

В настоящей работе численно изучено влияние полного покрытия внешней поверхности одностенных УНТ $(3,0)$ и $(4,0)$ атомарным водородом на энергетические характеристики дефектных конфигураций, возникающих в результате трансформаций SW. Установлено, что изменение этих характеристик при гидрировании УНТ определяется как типом УНТ, так и ориентацией поворачивающейся при трансформации SW связи C-C относительно оси УНТ.

При повороте параллельной оси УНТ связи С-C энергия негидрированной УНТ $(3,0)$ понижается, т.е. конфигурация с дефектом SW является устойчивой, в то время как энергия негидрированной УНТ $(4,0)$, напротив, возрастает, т.е. у этой УНТ устойчивая конфигурация - бездефектная. Гидрирование УНТ $(3,0)$ приводит к тому, что устойчивой также становится бездефектная конфигурация, тогда как после гидрирования УНТ $(4,0)$ бездефектная конфигурация остается устойчивой, а энергетические барьеры для образования и отжига дефекта SW повышаются.

Энергии конфигураций, образующихся при повороте направленной под углом к оси УНТ связи $\mathrm{C}-\mathrm{C}$, в обеих негидрированных УНТ $(3,0)$ и $(4,0)$ больше энергий соответствующих исходных конфигураций. После гидрирования УНТ бездефектные конфигурации остаются устойчивыми, а барьеры для образования и отжига дефектов SW либо существенно понижаются, либо изменяются крайне незначительно.

При гидрировании механическая жесткость обеих УНТ уменьшается. Дефекты SW приводят к уменьшению жесткости как негидрированных, так и гидрированных УНТ.

\section{Список литературы}

[1] H.W. Kroto, J.R. Heath, S.C. O’Brien, R.F. Curl, R.E. Smalley. Nature 318, 162 (1985).

[2] S. Iijima. Nature 354, 56 (1991).

[3] K.S. Novoselov, A.K. Geim, S.V. Morozov, D. Jiang, Y. Zhang, S.V. Dubonos, I.V. Grigorieva, A.A. Firsov. Science 306, 666 (2004).

[4] A.J. Stone, D.J. Wales. Chem. Phys. Lett. 128, 501 (1986).

[5] B.R. Eggen, M.I. Heggie, G. Jungnickel, C.D. Latham, R. Jones, P.R. Briddon. Science 272, 87 (1996).

[6] Q. Lu, B. Bhattacharya. Nanotechnology 16, 555 (2005).

[7] L. Pan, Z. Shen, Y. Jia, X. Dai. Physica B 407, 2763 (2012).

[8] D. Tasis, N. Tagmatarchis, A. Bianco, M. Prato. Chem. Rev. 106, 1105 (2006).

[9] C. Wang, G. Zhou, H. Liu, W. Duan. J. Phys. Chem. B 110, 10266 (2006).

[10] R. Ansari, S. Ajori, B. Motevalli. Superlat. Microstr. 51, 274 (2012).

[11] S.N. Shirodkar, U.V. Waghmare. Phys. Rev. B 86, 165401 (2012).

[12] С.С. Моливер, Р.Р. Зимагуллов, А.Л. Семенов. Письма в ЖТФ 37, 68 (2011).

[13] А.И. Подливаев, Л.А. Опенов. ФТТ 60, 160 (2018).

[14] J.-Y. Yi, J. Bernholc. Chem. Phys. Lett. 403, 359 (2005).

[15] A.J.M. Nascimento, R.W. Nunes. Nanotechnology 24, 435707 (2013).

[16] А.И. Подливаев, Л.А. Опенов. ФТТ 57, 2485 (2015).

[17] D. Stojkovic, P. Zhang, V.H. Crespi. Phys. Rev. Lett. 87, 125502 (2001).

[18] M. Kabir, K.J. Van Vliet. J. Phys. Chem. C 120, 1989 (2016).

[19] G.V. Vineyard. J. Phys. Chem. Solids 3, 121 (1957).

[20] M.M. Maslov, A.I. Podlivaev, K.P. Katin. Molecular Simulation 42, 305 (2016).

[21] Л.А. Опенов, А.И. Подливаев. ФТТ 50, 1146 (2008).

[22] K.P. Katin, V.S. Prudkovskiy, M.M. Maslov. Physica E 81, 1 (2016).

[23] Л.А. Опенов, А.И. Подливаев. Письма в ЖЭТФ 104, 192 (2016).

[24] M.M. Maslov, K.P. Katin. Chem. Phys. Lett. 644, 280 (2016).

[25] L.G. Zhou, S.-Q. Shi. Appl. Phys. Lett. 83, 1222 (2003).

[26] L.-M. Peng, Z.L. Zhang, Z.Q. Xue, Q.D. Wu, Z.N. Gu, D.G. Pettifor. Phys. Rev. Lett. 85, 3249 (2000).

[27] T.C. Fitzgibbons, M. Guthrie, E. Xu, V.H. Crespi, S.K. Davidowski, G.D. Cody, N. Alem, J.V. Badding. Nature Mater. 14, 43 (2015).

[28] R.E. Roman, K. Kwan, S.W. Cranford. Nano Lett. 15, 1585 (2015).

[29] C.D. Reddy, S. Rajendran, K.M. Liew. Nanotechnology 17, 864 (2006)

[30] E. Hernández, C. Goze, P. Bernier, A. Rubio. Phys. Rev. Lett. 80, 4502 (1998).

[31] О.Е. Глухова, О.А. Терентьев. ФТТ 48, 1329 (2006).

[32] M.M.J. Treacy, T.W. Ebbesen, J.M. Gibson. Nature 381, 678 (1996). 